\title{
Stellate Ganglion Block for Treatment of Ischemia and Pain Associated with Autolmmune Associated Raynaud's Syndrome
}

\author{
Matthew J. Kortes, MD${ }^{1}$, Whitney Luke, MD ${ }^{1,2}$, Ryan Thompson, MD $^{1}$, and Matthew J. Gray, MD ${ }^{1}$
}

The etiology of Raynaud's syndrome is characterized by inappropriate vascular constriction of digits and extremities from a complex interaction between the sympathetic nervous system, vascular endothelium, and the paracrine/endocrine system. Secondary Raynaud's, complicated by autoimmunity, is generally more severe and was not expected to respond to sympathetic blocks. Our report demonstrates profound improvement of angiographically confirmed severe secondary Raynaud's following stellate ganglion block, with resolution of multiple nonhealing digital ulcers.

A 53-year-old woman with mixed connective tissue disease and idiopathic thrombocytopenic purpura (ITP) presented with right hand pain, severe ischemic changes, and several areas of nonhealing digital ulcerations. Maximal medical management had provided transient symptomatic improvement. When angiographic findings of diffuse vasospasm on the right suggested increased sympathetic tone, we performed a trial of stellate ganglion blocks. The first block immediately improved vasoconstriction and pain. Over 7 months she underwent three series of weekly injections (11 total injections). Slow healing of her ulcerations occurred. At 6-month follow-up, her ulcerations remained healed.

Prior reports had indicated stellate ganglion or sympathetic blocks are ineffective or contraindicated in secondary Raynaud's. The resolution of dry gangrene resulting in digit salvage after serial stellate ganglion blocks suggests that increased sympathetic tone may be a mechanism of disease in secondary Raynaud's. Despite prior reports to the contrary, some patients may benefit from a trial of sympathetic blocks before undergoing surgical sympathectomy.

Key words: Raynaud's syndrome, mixed connective tissue disorder, stellate ganglion block, peripheral ischemia, secondary Raynaud's, sympathectomy
Raynaud's syndrome is a disease of inappropriate sympathetic tone resulting in a local vasospasm of elements of the arterial network often including the arterioles, arterial venous anastomoses, and sometimes more proximal vessels (1). Raynaud's is subdivided into primary Raynaud's, which is a relatively

From : ${ }^{1}$ Department of Physical Medicine and Rehabilitation, The Ohio State University Wexner Medical Center; ${ }^{2}$ The Ohio State University James Cancer Center and Solove Research Institute

Author for correspondence:Whitney Luke, MD

Address: The Ohio State University Wexner Medical Center, 480 Medical Center Drive, 1018 Dodd Hall, Columbus, OH 43210

E-mail: whitney.luke@osumc.edu common phenomenon and not associated with an underlying disease, and secondary Raynaud's, which is caused by underlying systemic disease. Secondary Raynaud's is often more severe and generally requires pharmacological treatment (2). A number of diseases are associated with secondary Raynaud's, including mixed connective tissue disease (MCTD), scleroderma, systemic lupus erythematosus and other autoimmune diseases. When pharmacological treatment is needed, exploration of secondary cause is advocated (3).

Treatment of secondary Raynaud's is typically based on severity and occurs in a step-wise fashion. For more moderate symptoms that require phar- 
macological therapy, a calcium channel blocker or selective serotonin reuptake inhibitor (SSRI) is often trialed first $(2,4)$. Failing an adequate response or tolerability, phosphodiesterase inhibitors, losartan or topical nitrate are several possible second-line treatments (3). For severe symptoms with ischemia, combination therapy including the use of anti-platelet agents is recommended. If symptoms fail to respond to combination therapy, prostaglandins, endothelin receptor inhibitors and/or botulinum toxin may be trialed before surgical sympathectomy is performed.

While often effective, a surgical sympathectomy presents a relatively high risk of complication and is irreversible even if the primary pathology may eventually be controlled. Surgical treatments either require microsurgery of the hand if the effects of the sympathectomy are to be limited (5), or thoracic surgery if the symptoms are more widespread (6). Both approaches have clear limitations, and while a number of interventional techniques have been reported, most of the evidence comes from case series or case reports. Here we present the case of a MCTD and coagulopathy-associated secondary Raynaud's syndrome with ischemic digital ulceration with early dry gangrene which was successfully treated by serial stellate ganglion blocks.

\section{CASE PRESENTATION}

A 53-year-old woman with complex past medical history of MCTD associated with pulmonary hypertension on sildenafil, idiopathic thrombocytopenic purpura (ITP), coagulopathy with 2 unprovoked lower limb DVTs, and Raynaud's syndrome presented as a referral for ongoing right forearm and hand pain. She was referred from vascular medicine for management of her right upper extremity pain due to chronic ischemia and ulcerations of digits one and 3 , with prior ulceration on digit 2, resulting from secondary Raynaud's syndrome of 20 months' duration.

Her initial symptoms consisted of intermittent blanching followed by purplish discoloration and pain in the affected digits. This occurred reliably with cold temperatures, but also sometimes spontaneously. A month after symptom onset, she was started on topical nitroglycerine and maintained on her prior dose of sildenafil, $20 \mathrm{mg}, 3$ times daily. Despite this, she developed ulcerations of digits one through 4 .
Due to worsening symptoms refractory to conventional treatment, she underwent bilateral upper limb angiography that demonstrated decreased arterial flow bilaterally, consistent with diffuse arterial vasospasm. There was resulting minimal flow to the bilateral hands (Fig. 1A and B). She was started on cilostazol after angiography. Pentoxifylline was added with some improvement, but without resolution of the ulcerations on digits one and 3. Amlodipine was added, after which digit 2 healed, but the ulcers on the remaining digits continued unchanged. Fourteen months after onset, a recurrence of ITP required treatment with intravenous immunoglobulin and rituximab before remission was achieved. Following treatment with rituximab, she noted subjective worsening of ischemic symptoms over the next 3 months and was hospitalized for evaluation of chronic ischemia pain of the right hand. She was referred to us for interventional treatment options after discharge.

Her exam was remarkable for a cold right upper limb with ulceration at digits one and 3 (Fig. 2A and B). There was a partially healed eschar on digit one. Her left limb was without digital ulcerations, but was also cool to the touch. Based on her reported history of Raynaud's and the presence of possible diffuse vasospasm on angiogram, we elected to proceed with a series of right stellate ganglion blocks.

The stellate ganglion block was performed by an anterior approach with $8 \mathrm{~mL}$ of $0.25 \%$ bupivacaine. Using a posterior/anterior (PA) view, a 2.5 inch, 22-gauge needle was advanced to the right $\mathrm{C} 6$ uncinate process between the trachea and major vessels including carotid artery, staying cranial to the likely location of the vertebral artery at C7. Contrast dye confirmed needle location and absence of vascular uptake before injection (Fig. 3).

After confirmation of location and the absence of vascular uptake, the injection was carried out with intermittent aspiration every $2 \mathrm{~mL}$. Immediately thereafter, she developed a transient Horner's syndrome lasting less than an hour, and the ulcerations on digits one and 3 began to bleed. Pain score improved from numeric rating scale (NRS) 7/10 before the procedure to $4 / 10,30$ minutes after.

The procedure was repeated weekly for four weeks with progressive increase in duration of Horner's sign 


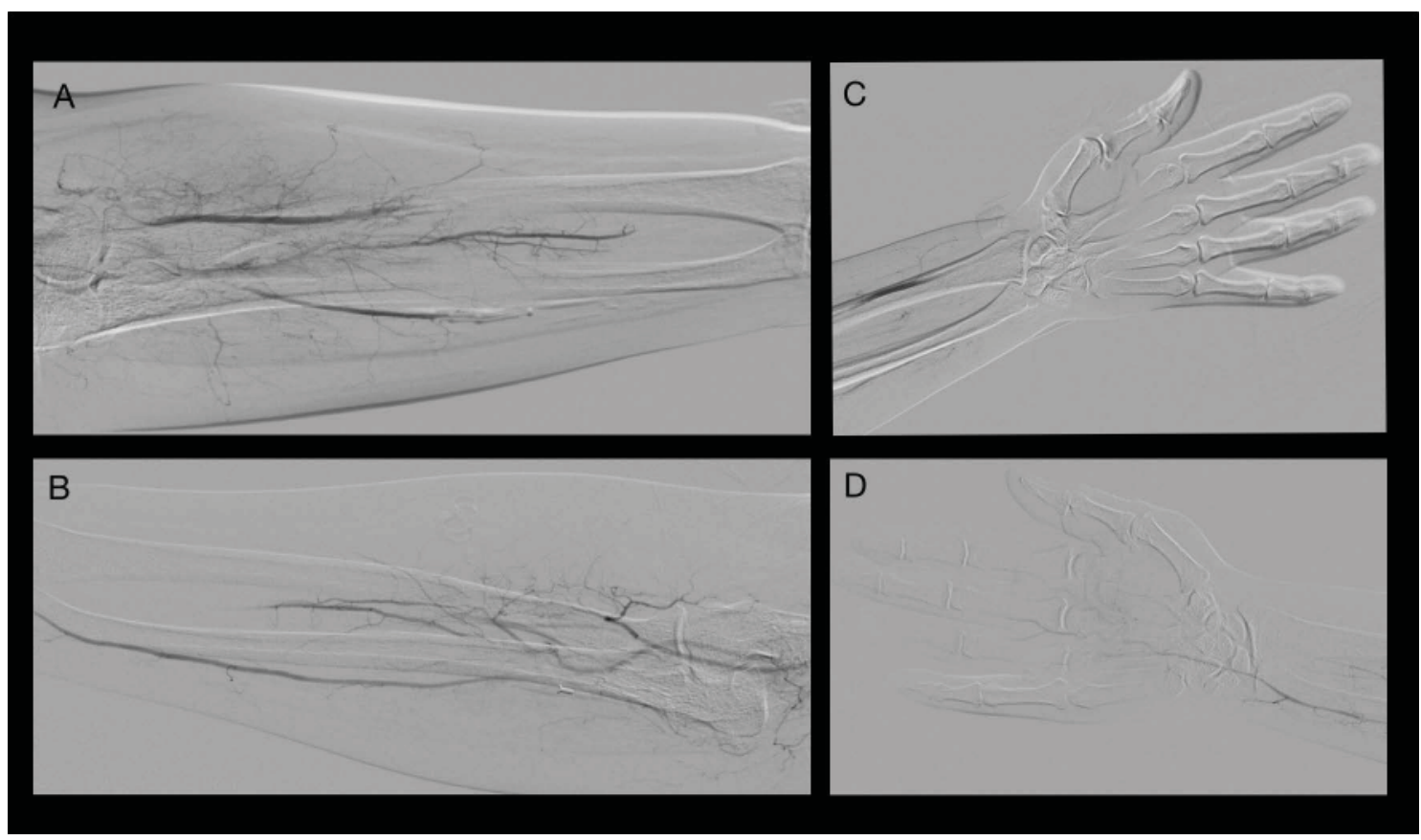

Fig. 1. A) Angiography of the left upper limb demonstrates no flow of contrast beyond the wrist in the distal left upper wrist. More proximally, the presence of narrow diameter radial, ulnar, and interosseus arteries with minimal flow is also present. B) Right upper limb with findings similar to the left side including minimal flow in the radial, ulnar and interosseus arteries. C) Left hand with very minimal arterial flow is seen. D) Right hand with only flow from the ulnar artery into the hand without flow from the radial artery.

to several hours and increased duration of pain relief between sequential blocks. By completion of the series, pain scores remained relatively stable at 3/10.

Two months later, she presented due to recurrence of pain, concerning for digital ischemia, but had maintained improvement of the digital ulcerations from prior to the initial block series. A series of 4 weekly blocks was repeated, with similar improvement in pain and digital perfusion starting after the first block and improving throughout the series. At scheduled follow-up 3 months later, ulceration at digit 3 was fully healed and digit one was partially healed. Given the improvement she reported after the previous series, a third series of 3 weekly stellate ganglion blocks was performed. At follow-up one month after completion of 11 total stellate ganglion blocks, the third digit was fully healed, and digit one was nearly healed with good tissue bed without eschar (Fig. 4). At follow-up 6 months after completion of the series, there was complete healing of ulcerations with reduction of average pain by $70 \%$, (average NRS was 1-2/10 from 7/10 prior to intervention).

\section{DISCUSSION}

This patient's experience suggests that stellate ganglion blocks can be a useful interventional treatment option to address symptoms of pain and digital ulceration associated with secondary Raynaud's syndrome, despite the likely contribution of multiple underlying disease states, when medication management has failed. Our patient was unusual for 2 key reasons. First, despite multiple factors affecting the development of secondary Raynaud's, the diagnostic workup demonstrated an underlying physiological abnormality contributing to her symptoms. Secondly, her treatment course may provide useful information to other clinicians when considering the use of stellate ganglion blocks to treat secondary Raynaud's (1-4,7). 


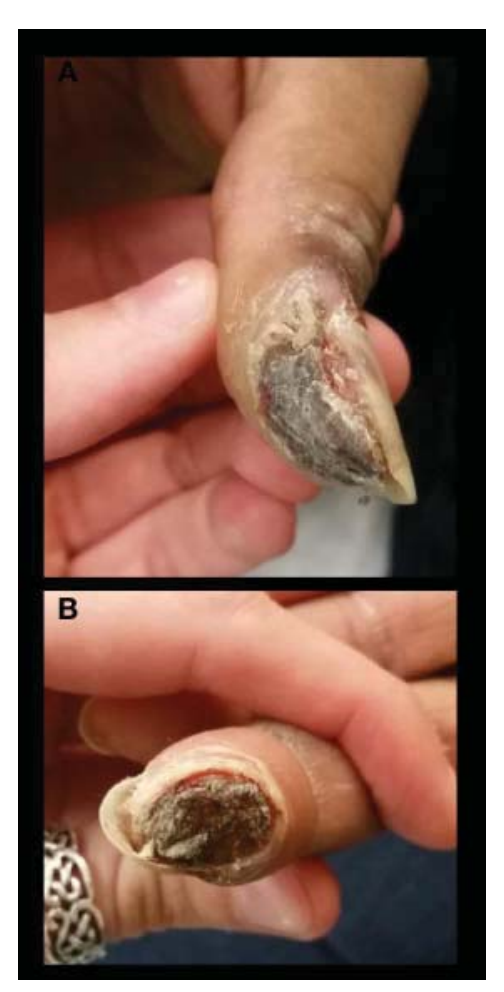

Fig. 2. A) Images prior to stellate ganglion block demonstrate ulceration and early dry gangrene of the right thumb.

B) Right index finger.

This patient has multiple factors which are known to be causative or associated with secondary Raynaud's. The presence of Raynaud's is exceptionally common and part of the diagnostic criteria for MCTD. While our patient's Raynaud's is most likely due to MCTD, coagulopathy and idiopathic thrombocytopenia likely influenced the severity of the symptoms (3). Despite digital ulceration being less common in MCTD than scleroderma, a sizable proportion of patients still develop digital ulceration and Raynaud's due to MCTD (8). It is likely that the presence of a hematological disorder resulted in an increased risk of ulceration, but angiography demonstrated diffuse bilateral vasospasm as the primary physiological cause of her disease.

The presence of demonstrated arterial vasospasm as the cause in this patient is important for several reasons. First, the improvement of symptoms after stellate ganglion block provides confirmation of the physiological cause of her secondary Raynaud's.
Specifically, it indicates that increased sympathetic tone in our patient led to the bilateral upper limb arterial vasospasm. This supports the use of sympathetic blocks in other patients with secondary Raynaud's when arterial vasospasm is present on angiography. Second, it demonstrates that some cases of secondary Raynaud's may have nuanced pathophysiological differences with treatment implications. This is important, as some cases of secondary Raynaud's may not respond to sympathetic blockade and in systemic scleroderma, symptoms may reportedly worsen (9).

A number of surgical procedures have been described for treatment of Raynaud's. Surgical sympathectomy represents the principal surgical approach to Raynaud's. In review, an aggregated $81 \%$ of patients with digital ulcers were found to improve after thoracic sympathectomy at T2-T4 (6). An endoscopic approach has been advocated (10); however, peri-arterial sympathectomy represents 
an alternative to thoracic sympathectomy and has been investigated in hopes of developing a surgical approach with less potential for serious morbidity. In patients with autoimmune disease, a similar percentage of long-term ulcer improvement was found with $75 \%$ of digits healing (5). Despite this, less invasive surgical treatment options continue to be sought.

Less invasive fluoroscopically guided percutaneous interventions have been minimally investigated for improvement of digital ulceration. Sympathetic chain ablations have been studied, with comparison between radiofrequency lesioning at T2/T3 or phenol injections. Outcome differences were minimal, as only 3 of 50 patients had ischemic lesions and improvement in digital ulceration was not the main outcome of the study (11).

Use of reversible regional and autonomic blocks has also been reported. Previously, stellate ganglion blocks have been trialed in patients with Raynaud's due to systemic scleroderma with mixed results $(9,12)$. However, several cases reporting the improvement of symptoms after regional anesthetic blocks with either lipophilic bupivacaine (13) or continuous infusion via catheter (14) support the potential of the use of nonablative treatments. Our case further supports the potential of nonablative treatments to address digital ulceration and pain in secondary Raynaud's.

Recently algorithms on the treatment of Raynaud's disease have been published, with most including surgical sympathectomy for refractory symptoms. Given the high morbidity rate and potential risk of mortality associated with thoracic sympathectomy, not all patients are medically able or willing to undergo it. In medically complex patients at high risk of surgical complication, a trial of stellate ganglion block prior to undergoing surgical sympathectomy could be appropriate.

This case differs from previously published cases because most reported cases were in patients not heavily pretreated with multiple usually effective medications (calcium channel blockers, phosphodiesterase inhibitors, pentoxifylline, etc.), who did not have evidence of diffuse vasoconstriction on angiography consistent with sympathetic over activation and did not undergo stellate ganglion block as a last resort prior to likely amputation, which was the case in our patient. Despite its utility in this case,

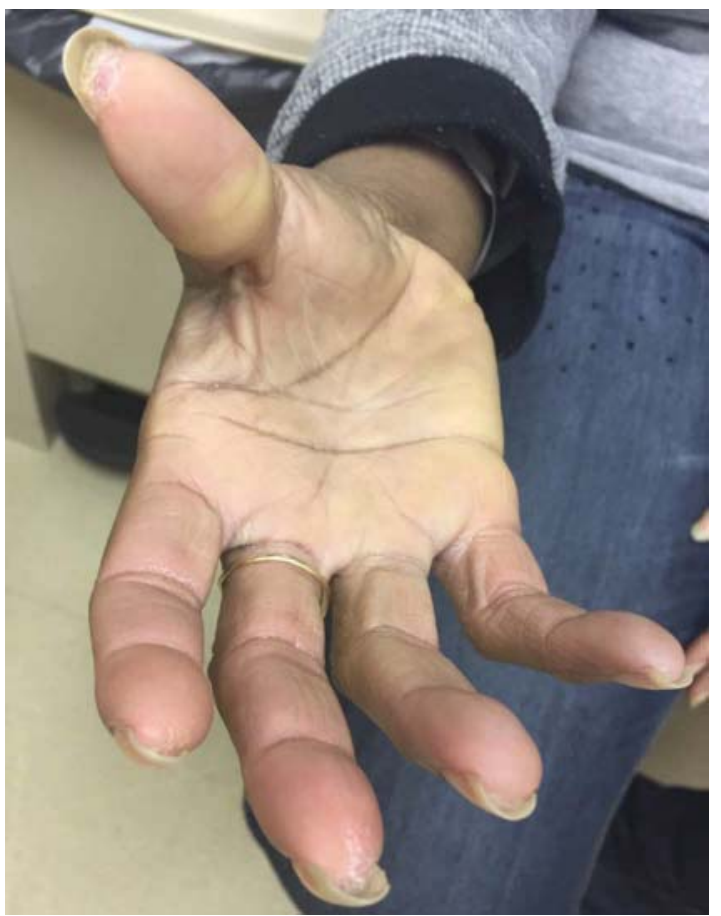

Fig. 4. Demonstrates right thumb and digits 2 through 5 after 12 stellate ganglion injections. The right thumb (A) is nearly healed, while the index finger has (B) good peripheral blood flow, good underlying tissue, and perfusion without eschar.

stellate ganglion block was not included as a possible treatment option prior to amputation in several well publicized algorithms.

\section{CONCLUSION}

Despite the bulk of surgical treatments targeting the sympathetic nervous system, the use of stellate ganglion blocks to address Raynaud's has remained under investigated and underutilized. Here, we demonstrate that in the presence of angiographyconfirmed arterial vasospasm, our patient's pain and digital ulceration improved after treatment with serial stellate ganglion blocks. Prior reports of worsening symptoms in scleroderma-associated secondary Raynaud's may not be generalizable to other causes of secondary Raynaud's.

Therefore, stellate ganglion block warrants more rigorous investigation as a treatment for secondary 
Raynaud's. Furthermore, in patients with digital ulceration who have failed multiple oral treatments, a trial of stellate ganglion blocks with close monitoring of symptoms immediately afterwards could be beneficial.

\section{REFERENCES}

1. Bakst R, Merola JF, Franks AG, Jr, Sanchez M. Raynaud's phenomenon: Pathogenesis and management. J Am Acad Dermatol 2008; 59:633-653.

2. Hughes M, Herrick AL. Raynaud's phenomenon. Best practice $Q$ research. Clinical Rheumatol 2016; 30:112-132.

3. Wigley FM, Flavahan NA. Raynaud's phenomenon. N Engl J Med 2016; 375:556-565.

4. Landry GJ. Current medical and surgical management of Raynaud's syndrome. J Vasc Surg 2013; 57:1710-1716.

5. Hartzell TL, Makhni EC, Sampson C. Long-term results of periarterial sympathectomy. J Hand Surg Am 2009; 34:1454-1460.

6. Coveliers HM, Hoexum F, Nederhoed JH, Wisselink W, Rauwerda JA. Thoracic sympathectomy for digital ischemia: A summary of evidence. J Vasc Surg 2011; 54:273-277.

7. Botzoris V, Drosos AA. Management of Raynaud's phenomenon and digital ulcers in systemic sclerosis. Joint Bone Spine 2011; 78:341-346.

8. Venables PJ. Mixed connective tissue disease. Lupus 2006; 15:132-137.

\section{Acknowledgment}

The authors are grateful to Beth at The Medical Wordsmith for her assistance in the preparation and submission of this manuscript.

9. Omote K, Kawamata M, Namiki A. Adverse effects of stellate ganglion block on Raynaud's phenomenon associated with progressive systemic sclerosis. Anesth Analg 1993; 77:1057-1060.

10. Matsumoto Y, Ueyama T, Endo M, Sasaki H, Kasashima F, Abe Y, Kosugi I. Endoscopic thoracic sympathectomy for Raynaud's phenomenon. J Vasc Surg 2002; 36:57-61.

11. Gabrhelik T, Michalek P, Adamus M, Berta E. Percutaneous upper thoracic radiofrequency sympathectomy in Raynaud phenomenon: A comparison of T2/T3 procedure versus T2 lesion with phenol application. Reg Anesth Pain Med 2009; 34:425-429.

12. Klyscz T, Junger M, Meyer H, Rassner G. Improvement of acral circulation in a patient with systemic sclerosis with stellate blocks. VASA. Zeitschrift fur Gefasskrankheiten 1998; 27:39-42.

13. Soberon JR, Duncan SF, Sternbergh WC. Treatment of digital ischemia with liposomal bupivacaine. Case Rep Anesthesiol 2014; 2014:853243.

14. Dao T, Amaro-Driedger D, Mehta J. Successful treatment of Raynaud's syndrome in a lupus patient with continuous bilateral popliteal sciatic nerve blocks: A case report. Local Reg Anesth 2016; 9:35-37. 